Original Paper http://ajol.info/index.php/ijbcs http://indexmedicus.afro.who.int

\title{
Caractérisation des dégâts provoqués par la faune sauvage à la périphérie de la Forêt des Marais Tanoe-Ehy au sud-est de la Côte d'Ivoire
}

\author{
Marthe Lydie KOUAO ${ }^{1,3 *}$, Jean-Claude Koffi BENE², André Djaha \\ $\mathrm{KOFF}^{2,3}$, Bertin Akpatou KOUAME ${ }^{1,3}$ et Inza KONE ${ }^{1,3}$ \\ ${ }^{1}$ Laboratoire de Zoologie et Biologie Animale, Université Félix Houphouët Boigny 22 BP 582 Abidjan \\ 22, Côte d'Ivoire. \\ ${ }^{2}$ Unité de Formation et de Recherches en Environnement, Université Jean Lorougnon Guédé, Daloa, \\ Côte d'Ivoire, BP 150 Daloa. \\ ${ }^{3}$ Centre Suisse de Recherches Scientifiques en Côte d'Ivoire (CSRS), 01 BP 1303 Abidjan 01, \\ Côte d'Ivoire. \\ *Auteur correspondant ; E-mail : martlydi@yahoo.fr; Tel: +22507414429/01695302
}

\section{RESUME}

En Côte d'Ivoire, les conflits hommes faunes sauvages existent. Cependant, en dehors de ceux portant sur les hommes et les éléphants, très peu de travaux scientifiques traitent de ce sujet. La principale cause connue de ces conflits semble être l'extension de l'agriculture à proximité des espaces protégés. Pour la mise en place d'une bonne stratégie de conservation de la FMTE, cette étude se propose de caractériser les dégâts provoqués par la faune à la périphérie de cette forêt. L'étude a consisté en une enquête dans quatre villages pour recueillir des informations sur les dégâts que cause la faune sur les biens des villageois. Ensuite, des prospections ont été organisées dans les champs périphériques de la FMTE à travers des grilles de dimensions 1000x500m, pour confirmer et évaluer l'ampleur de ces dégâts. De cette étude, il ressort que les animaux constituent des déprédateurs des différentes cultures pratiquées par les populations. De plus, diverses espèces animales sont impliquées dans ces déprédations. Parmi celles-ci, les singes et les aulacodes provoquent de grandes pertes aux planteurs. Les méthodes adoptées pour se prémunir des incursions, semblent malheureusement être inefficaces.

(C) 2018 International Formulae Group. All rights reserved.

Mots clés : conflit homme-faune,cultures, animaux sauvages, déprédateurs, conservation, Forêt des Marais Tanoé-Ehy

\section{Wildlife crop damage characterization around Forêts des Marais Tanoe- Ehy, south-east in Côte d'Ivoire}

\begin{abstract}
In Côte d'Ivoire, wildlife-human conflicts exist. Apart from human-elephants conflicts, very little scientific work deals with this subject. The main cause of these conflicts seems to be the extension of agriculture close to protected areas. To implement a good conservation strategy around FMTE, this study seeks to characterize the damage caused by wildlife on forest boundary. Data collection was done in four villages to collect information on the damage caused by wildlife on the property of villagers. Then, samples were collected in the farms on boundary of FMTE through grids of dimensions 1000x500m, to confirm and
\end{abstract}


evaluate the extent wildlife damage. The study reveals diverse crop raiders of different crops. Among these, monkeys and cane rats cause great losses to peasants. The methods adopted to guard against incursions unfortunately seem to be ineffective.

(C) 2018 International Formulae Group. All rights reserved.

Keywords: Human-wildlife, crops, wildlife, crop raiders, conservation, Forêt des Marais Tanoé-Ehy.

\section{INTRODUCTION}

Les conflits homme-faune sauvage se rencontrent partout dans le monde entier (Hill, 2004) mais avec un pic en Afrique. Un conflit homme-faune se définit par toute interaction entre l'homme et les animaux sauvages avec des impacts négatifs sur la vie économique, sociale et culturelle des hommes et sur la conservation des espèces animales ou sur l'environnement (WWF, 2005). La principale cause directe de ces conflits semble être l'extension de l'agriculture (Bulte et Rondeau, 2007) à proximité des forêts délimitées pour la conservation durable de la biodiversité. Cette extension des terres agricoles en Afrique s'accroit pour répondre aux besoins alimentaires et financiers d'une démographie galopante (Kouelo et al., 2016; Zoumarou et al., 2016; Enbakom et al., 2017; Ye et al., 2017). Selon FAO (2010), la démographie africaine déjà croissante, va se poursuivre et la population doublera d'ici 2050. Les conflits homme-faune sauvage risquent alors de s'accentuer face à cette croissance qui sans doute entrainera l'installation massive des populations humaines aux abords des aires protégées à la recherche de terres disponibles.

Les conflits provoqués par les animaux se traduisent pour la plupart, par leur intrusion dans les zones d'habitation humaine, la destruction des cultures, leur attaque des provisions alimentaires et l'endommagement des sources d'eau ou d'autres structures humaines, tuant ou blessant par occasion les habitants (FAO, 2010). Diverses solutions allant de la surveillance à l'abattage des animaux de manière sélective (juste l'individu fautif) ou aléatoire (tout représentant de l'espèce problématique) (Marchand, 2013) sont adoptées par les humains pour se prémunir des attaque (Fairet, 2012).
En Côte d'Ivoire, la plupart des travaux relatifs aux conflits homme-faune sauvage concernent les interactions des hommes avec les éléphants (Atta et al., 2016). Dans ce pays, les éléphants font de plus en plus d'intrusions dans les cultures (cacao, café, hévéa, coco, palmier à huile, manioc, banane, etc.) suscitant le mécontentement des paysans (Atta, 2016). Cependant, depuis peu, le Ministère en charge des forêts communique de plus en plus sur la récurrence de conflit entre les hommes et une large gamme d'animaux sauvages en dehors de ceux impliquant les éléphants (MINEF, 2015). Fort heureusement, ces communications ont trouvé un écho favorable auprès de certains chercheurs, qui ont diligenté pour une première fois des travaux relatifs à la thématique autour de la Forêt des Marais Tanoé-Ehy (FMTE) (Sidawy, 2011). Les résultats de ces travaux ont suggéré une quantification des dégâts subis par les populations riveraines, d'où l'intérêt que notre étude porte sur la caractérisation des dégâts provoqués par la faune sauvage autour de la FMTE. La forêt des marais Tanoé-Ehy (FMTE), située au sud-est de la Côte d'Ivoire mérite le plus grand intérêt pour la conservation d'espèces de singes gravement menacées d'extinction (Koné et al., 2012) inscrites sur la liste rouge de l'UICN (Béné, 2013). Au même moment, des dégâts causés par les singes sont rapportés de manière répétitive à des chercheurs du Centre Suisse de Recherches Scientifiques en Côte d'Ivoire au cours de leurs différentes missions autour de la FMTE (Sidawy, 2011). Par ailleurs, il a été prouvé que les conflits homme-faune constituent un défi difficile à relever pour la conservation durable des forêts (Hill, 2004) qui offrent une série de biens et de services parmi lesquelles, la diversité biologique (Faye, 2018).

Cette étude vient ainsi fournir les 
informations sur les entités impliquées dans ce type de conflit, l'ampleur des pertes subies par les populations et les actions de l'homme pour se prémunir des dégâts provoqués par les animaux sauvages.

\section{MATERIEL ET METHODES Milieu d'étude}

La Forêt des Marais Tanoé-Ehy (FMTE), d'une superficie de 12.000 hectares est située à l'interface de plusieurs villages (Saykro, Ehania Tanoé, Kongodjan Tanoé, Kadjakro, Yao-Akakro, Atchimanou, Nouamou, Dohouan, Kotoagnuan). Elle est par ailleurs bordée par la rivière Tanoé et la lagune Ehy à l'extrême Sud-Est de la Côte d'Ivoire à la frontière avec le Ghana. Elle présente deux différents paysages. Un paysage de forêt dense proche des villages de Yao-Akakro, de Kongodjan Tanoé, de Kadjakro et un paysage de forêt dégradée proche des six autres villages restants (Figure 1). La FMTE se caractérise également par la richesse et la spécificité de sa faune et de sa flore (Kpan, 2011). Elle regorge en effet de nombreuses espèces endémiques à statut particulier en ce qui concerne les plantes, les poissons, les oiseaux, les amphibiens et les mammifères (Kpan, 2011). La population riveraine de cette forêt est composée par les autochtones Agni et Appolo (qui cohabitent de façon pacifique avec les allochtones Baoulé, Sénoufo, Malinké) et par des étrangers burkinabés, ghanéens et maliens. L'économie de la région est dominée par l'agriculture; ce qui a eu pour conséquence le remplacement des écosystèmes forestiers par de vastes plantations de cocotiers et de palmiers à huile dont certaines appartiennent aux villageois et d'autres à la Palmeraie Industrielle de Côte d'Ivoire (PALMCI) (Zadou et al., 2011). Les périphéries immédiates de la FMTE sont bordées par des champs de cultures vivrières (maïs, manioc principalement) et de cultures de rente (cacao, hévéa, palmiers à huile). Outre les cultures vivrières et de rente pratiquées, aux abords de la FMTE, une grande frange de la population pratique la pêche dans les nombreux cours d'eau qui parcourent la FMTE, notamment le fleuve Tanoé, la lagune Ehy et leurs ramifications.

\section{Matériel}

Le matériel qui a servi à l'étude se compose de fiches d'enquêtes, d'un GPS (Global Positioning System) de type GARMIN qui a servi à circonscrire les surfaces de cultures et en prendre leurs coordonnées géographiques, un ruban gradué de $50 \mathrm{~m}$, pour délimiter les grilles d'observation aux périphéries immédiates du site forestier, un guide des mammifères pour l'identification des animaux sauvages. Le matériel biologique est constitué par les plantes agricoles, tous les animaux susceptibles d'être rencontrés sur le site d'étude et l'ensemble des indices de présence à caractère biologique (restes alimentaires, crottes, empreintes, etc...).

\section{Méthodes}

La collecte des données s'est déroulée en deux phases. La première a concerné les enquêtes quantitatives et la deuxième phase a consisté à des observations directes dans les grilles virtuelles disposées sur le terrain.

\section{Enquête quantitative}

Le questionnaire administré a été calqué sur celui proposé par le groupe des spécialistes des éléphants d'Afrique autour des conflits homme-éléphants (Parker et al., 2007).

Sur les neuf villages autour de la FMTE, en rapport avec le type de paysages que présente la forêt, quatre ont été choisis pour cette étude en raison de deux villages du côté de chaque paysage «forêt dense et forêt dégradé ». Les villages de Yao-Akakro et Kongondjan-Tanoé sont du côté de la forêt dense et ceux de Dohouan et de Kotoagnoan sont du côté de la forêt dégradée (Figure 1). Ces villages ont été parcourus et les populations riveraines ont été interrogées sur la thématique des conflits homme faune. La taille de l'échantillon a été obtenue à partir de la formule: $\mathrm{n}=\mathrm{t}^{2}$. p.(1-P) / $\mathrm{e}^{2}$ (Pupion, 2008). $\mathrm{n}$ représente la taille de la population totale à interroger, $t$ représente le niveau de confiance (selon la loi normale centrée réduite) fixé à $95 \%$ (sa valeur est égale à 1,96); p représente la prévalence des confits homme faune dans la zone du projet. La prévalence $\mathrm{p}$ n'étant pas connue, l'on considère $p$. $(1-p)=0,25$; e est la marge d'erreur fixée à $5 \%$. 
Après application de la formule, l'on a obtenu $\mathrm{n}=103$. Sur ces 103 personnes à interroger au total, le nombre d'enquêtés par village a été réparti proportionnellement à la taille de la population par rapport au recensement général de la population et de l'habitat de 2014. Ainsi, 16 villageois ont été interrogés à Yao-akakro, 16 autres à Kongodjan Tanoe, 17 à Dohouan et 54 pour le gros village de Kotouagnoan.

\section{Observations directes dans les champs}

Pour la collecte des données, deux assistants de terrain notamment des anciens chasseurs convertis à la protection de la faune à la suite de différentes formations en la matière ont été recrutés dans chaque village.

$\begin{array}{cccr}\begin{array}{c}\text { Etablissement } \\ \text { d'observations }\end{array} & \text { des } & \text { grilles } & \text { virtuelles } \\ \text { La méthode } & \text { utilisée pour }\end{array}$
l'établissement des grilles, est celle utilisée par Naughton-Treves (1998) mais avec quelques adaptations devant la réalité du terrain. Elle a posé des grilles de $1000 \mathrm{~m}$ sur $500 \mathrm{~m}$ à une des limites du parc national de Kibale de six villages le long de laquelle sont retrouvés des champs à partir du bord du parc jusqu'à $500 \mathrm{~m}$. Elle a systématiquement quadrillé ces grilles par des mailles de $25 \mathrm{~m}^{2}$ à l'intérieur des champs. Dans le cadre de cette étude, des grilles virtuelles ont été disposées de manière aléatoire dans les champs se retrouvant à deux différentes limites de la FMTE suivant les critères ci-après : (1) la présence de champs sur $500 \mathrm{~m}$ à partir du bord de la forêt, (2) l'accord des paysans pour la visite de leurs champs, (3) la distance à parcourir du village pour atteindre ces champs à pieds. Les champs doivent être dans un rayon de $2 \mathrm{~km}$ du village afin de les rallier facilement à pieds. Sur ces critères, le village de Kotouagnoan a été écarté pour la suite des travaux après y avoir mené les enquêtes. La plupart des personnes interrogées ont indiqué que les premières parcelles à la limite de la forêt sont à plus de $2 \mathrm{~km}$ de ce village.

Les dimensions des grilles ont également été de $1000 \mathrm{~m}$ sur $500 \mathrm{~m}$, mais avec des mailles virtuelles représentées par les surfaces des différentes parcelles de cultures bien distinctes circonscrites avec le GPS dont les superficies sont exprimées en hectares (ha) (Tableau 1).

NB : Quelques relevés additionnels ont été faits hors des grilles mais au niveau des limites immédiates de la FMTE, sur la base des informations reçues lors des enquêtes.

\section{Estimation du nombre total de plants pour chaque culture}

Les prospections directes débutées en Avril, ont coïncidé avec la période de semis du maïs. Pour connaître le nombre total de plants/pieds de maïs sur chaque parcelle, nous en avons profité pour délimiter une surface de $25 \mathrm{~m}^{2}$ et y avons compté les trous creusés contenant les semis. Pour ce qui concerne Kongodjan, le début des prospections a débuté après la période de semis pour causes de difficultés liés au déplacement.

Les pieds de manioc ont été également sur une surface de $25 \mathrm{~m}^{2}$ hébergeant de jeunes plants de manioc. Considérant que les pieds de ces cultures sont répartis de façon homogène sur les parcelles, le nombre obtenu est extrapolé à toutes les parcelles visitées hébergeant l'une de ces deux cultures.

En ce qui concerne les cultures de rente, nous avons également comptés les pieds de cacaoyer sur une surface de $25 \mathrm{~m}^{2}$ dans l'un des champs de cacaoyers. Les pieds de palmiers à huile n'ont pas été comptés sur le terrain. Contrairement aux cacaoyers où les prescriptions quant au mode de repiquage sont contournées, les paysans ont été formels quant au respect des prescriptions laissées par la structure étatique en charge de la filière palmiers à huile.

\section{Estimation du nombre total de pieds/organes touchés par les animaux}

Pour estimer le nombre total de pieds et d'organes touchés, une marche lente de prospection à travers les parcelles de cultures a été faite. Au cours de cette marche, nous scrutons les plants de la racine aux feuilles afin de déterminer les plants touchés par les animaux et nous les comptons par parcelle (maille virtuelle) comme fait par Hill (2000) au cours de leurs travaux. Comme eux, nous avons noté sur chaque parcelle de cultures, le type de cultures, les traces laissées par les animaux sur des parties de la plante, le stade de 
développement des plants touchés par les animaux (Tableau 1). Enfin, nous avons enregistré l'état de la plante entière après déprédation, ainsi que les moyens utilisés pour se prémunir des attaques des animaux. Le cumul du nombre de pieds ou organes touchés à chaque visite a donné le nombre total de pieds ou organes touchés par chaque espèce animale sur chaque parcelle concernée. Le nombre total d'organes non directement visibles tels que les tubercules de manioc a été obtenu en comptant les tubercules mis à nus par les animaux sous trois pieds de manioc choisis au hasard. La moyenne obtenue a été ramenée à 1 pied de manioc. D'avril 2016 à janvier 2017, la collecte de données a été faite chaque deux semaines comme l'a fait Fairet (2012) pour son étude au sujet des conflits homme-faune autour du parc de Loango au Gabon.

\section{Analyse des données}

Pour les analyses, seulement les données d'une saison de cultures de maïs (qui part d'Avril à Juillet pour la première et de Septembre à Décembre pour la deuxième) ont été prises en compte. Les cultures de manioc visitées ont une durée de développement de six mois à 1 an, les mêmes parcelles ont servi pour toute la durée de l'étude. Pour les parcelles de cacaoyers, l'estimation des pertes a été faite avant la récolte des cabosses.

La comparaison de l'étendue des dégâts dans les parcelles de cultures dans les différents villages a été faite par le test non paramétrique de Kruskal-wallis. Les analyses statistiques ont été réalisées avec le logiciel SPSS.20.

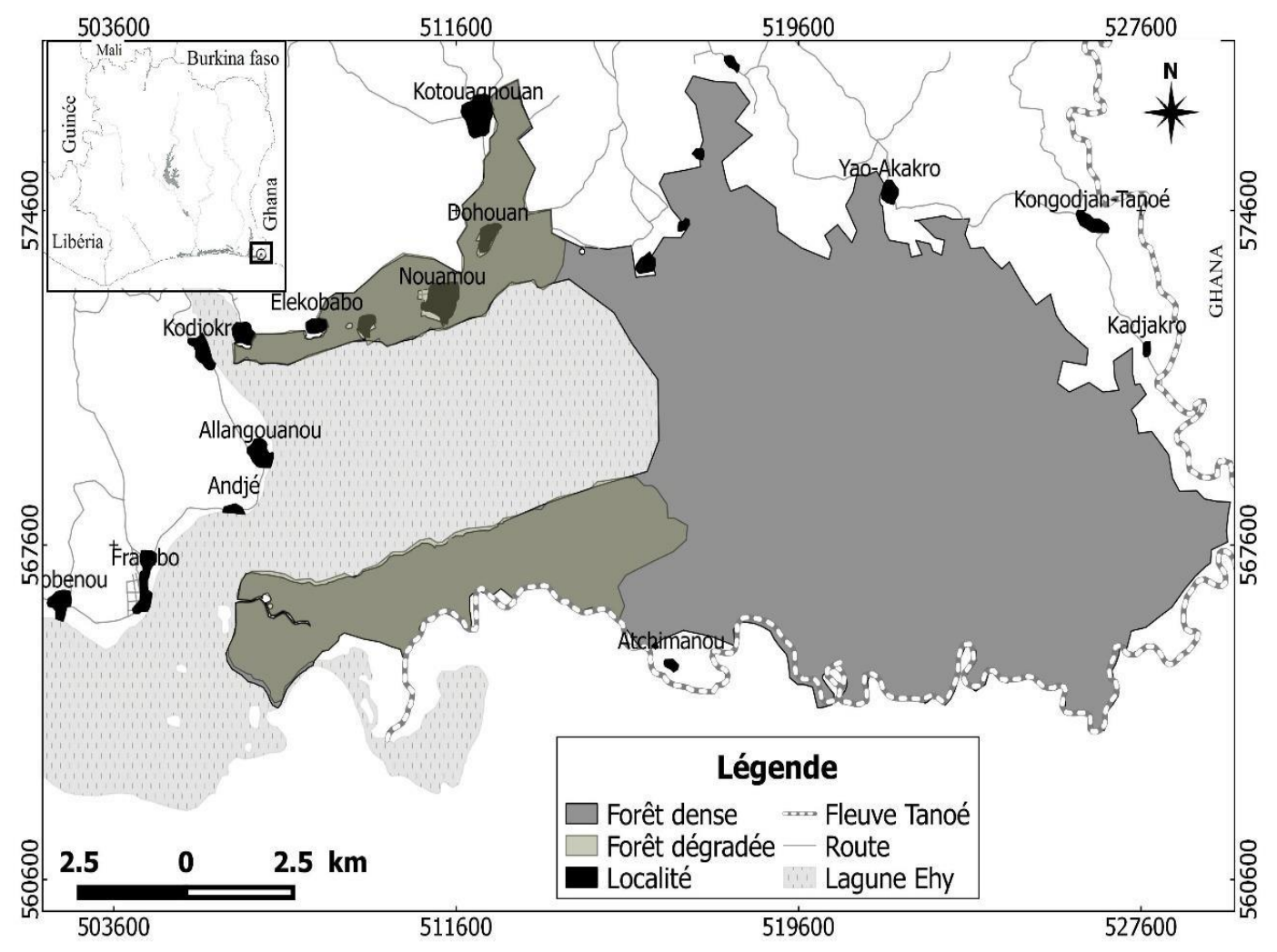

Figure 1: Localisation de la zone d'étude (RASAPCI, 2010). 
Tableau 1 : Surfaces des cultures échantillonnées et leurs stades de développement au début des prospections par village.

\begin{tabular}{|c|c|c|c|c|}
\hline Villages & Cultures & $\begin{array}{c}\text { Nombre } \\
\text { de } \\
\text { parcelles } \\
\text { visitées }\end{array}$ & $\begin{array}{c}\text { Surfaces } \\
\text { totales visitées } \\
\text { (ha) }\end{array}$ & $\begin{array}{l}\text { Stades de développement des } \\
\text { premières observations }\end{array}$ \\
\hline \multirow{3}{*}{ Dohouan } & Maïs (Zea mays) & 6 & 4,43 & Semis \\
\hline & $\begin{array}{l}\text { Manioc ** (Manihot } \\
\text { esculenta })\end{array}$ & 1 & 1 & Jeunes plants \\
\hline & $\begin{array}{l}\text { Palmiers (Elaeis } \\
\text { guineensis) }\end{array}$ & 1 & 1 & Jeunes plants/ plants matures \\
\hline \multirow{5}{*}{ Kongodjan } & Maïs (Zea mays) & 5 & 1,26 & Jeunes plants \\
\hline & $\begin{array}{c}\text { Manioc ** (Manihot } \\
\text { esculenta) }\end{array}$ & 7 & 2,135 & Jeunes plants/plants matures \\
\hline & $\begin{array}{l}\text { Palmiers (Elaeis } \\
\text { guineensis) }\end{array}$ & 1 & 1 & Jeunes plants \\
\hline & $\begin{array}{l}\text { Cacao (Theobroma } \\
\text { cacao) }\end{array}$ & 2 & 2 & Plants matures \\
\hline & $\begin{array}{l}\text { Bananiers (Musa } \\
\text { paradisiaca) }\end{array}$ & 1 & ND & Plants matures \\
\hline \multirow{6}{*}{ Yao-akakro } & Maïs (Zea mays) & 8 & 1,547 & semis \\
\hline & $\begin{array}{l}\text { Manioc**(Manihot } \\
\text { esculenta })\end{array}$ & 8 & 4,834 & Jeunes plants/plants matures \\
\hline & $\begin{array}{c}\text { Manioc**(Manihot } \\
\text { esculenta }) \mathrm{HG}\end{array}$ & $2^{2}$ & 0,0233 & \\
\hline & $\begin{array}{l}\text { Palmiers (Elaeis } \\
\text { guineensis) }\end{array}$ & 1 & 2,5 & Jeunes plants \\
\hline & $\begin{array}{c}\text { Cacao (Theobroma } \\
\text { cacao) }\end{array}$ & 1 & 1,5 & Plants matures \\
\hline & $\begin{array}{l}\text { Taro (Colocasia } \\
\text { esculenta) }\end{array}$ & 1 & ND & Plants matures \\
\hline
\end{tabular}

Légende : $\mathrm{N} \mathrm{D}=$ Non disponible. Les pieds de ces spéculations n'ont pas été convertis en superficies, un pied de bananier et deux pieds de taro ont été retrouvés séparément sur deux différentes parcelles parsemées de manioc.

Manioc** = variété foutou (Manihot $\mathrm{sp})$ et variété gari (Manihot $\mathrm{sp})$ avec une nette prépondérance des pieds variété gari/attiéké sur les parcelles.

HG1 = prélèvement sur le dispositif hors grilles, une seule incursion y a été relevée. 


\section{RESULTATS}

Entités impliquées dans le conflit existant à la périphérie de la FMTE

Du côté des hommes

L'enquête a révélé deux catégories de personnes qui sont en conflit avec les animaux sauvages de la FMTE : les planteurs, pour $71 \%$ des enquêtés et les pêcheurs pour $29 \%$ d'entre eux.

\section{Du côté des animaux}

Plusieurs groupes zoologiques sont incriminés pour les dégâts subis par les planteurs et pêcheurs victimes aussi bien du côté forêt dégradée que du côté forêt dense (Figure 2). En ce qui concerne les dégâts sur les cultures, ce sont : l'aulacode, le guib harnaché, les écureuils, les singes, l'athérure africain, le rat géant. Pour ce qui concerne la pêche, ce sont : les loutres, les crocodiles, les tortues, les crabes.

\section{Nature des dégâts provoqués par les animaux sauvages \\ Cultures et autres biens privés}

Selon $52 \%$ des personnes interrogées, les cultures sont plus exposées aux incursions des animaux, selon $33 \%$ autres enquêtés, ce sont plutôt les produits et matériels de pêches qui sont exposés. Mis à part les avis par ces deux catégories de personnes, 3\% des 103 de l'échantillon pensent que les animaux d'élevage sont plus menacés par la présence de certains animaux sauvages dans les environs, $1 \%$ pensent que les animaux s'attaquent plus au grenier et les $11 \%$ restants ont refusé de se prononcer sur le sujet.

\section{Blessures/mort d'hommes}

Sur les 103 personnes interrogées, aucune personne n'a affirmé avoir été témoin d'agressions ou de morts d'hommes provoquées par un animal sauvage de la FMTE.

\section{Identification des espèces animales déprédatrices \\ Suivant les enquêtes}

Du côté de la forêt dégradée à Dohouan, les animaux les plus incriminés en ce qui concerne les cultures, sont : les aulacodes, selon $93 \%$ des enquêtés suivis des potamochères pour $73 \%$ des mêmes enquêtés (Figure 2). En ce qui est de la pêche, les crocodiles et les loutres ont été accusés pour chacun de ces groupes zoologiques par $67 \%$ des enquêtés.

A Kotouagnoan, les aulacodes ont été plus incriminés par $78 \%$ des enquêtés suivis des guib, par $61 \%$ des mêmes enquêtés.

Du côté de la forêt dense à KongodjanTanoé, les aulacodes ont été incriminés par toutes les personnes interrogées $(100 \%)$, suivis des primates $(75 \%$ des personnes interrogées). En ce qui est de la pêche, les crocodiles ont été rendus coupables par $69 \%$ des personnes interrogées (Figure 2).

A Yao-Akakro, les animaux les plus cités sont les athérures, 60\% des enquêtés, suivis des aulacodes. Les loutres et les crocodiles sont accusés pour chacun de ces groupes zoologiques par 7\% des populations approchées (Figure 2).

\section{Suivant les observations directes}

Après échantillonnage dans les parcelles de cultures, Du côté de la forêt dégradée au niveau de Dohouan, nous notons que les grands déprédateurs sont les aulacodes Thryonomys swinderianus, qui ont causé la perte de de $9 \%$ de jeunes pieds de palmier sur la parcelle hébergeant cette culture (Tableau 2).

Du côté de la forêt dense, en ce qui concerne Kongodjan, les plus gros ravageurs de cultures sont les cercopithèques Cercopithecus sp., suivis des aulacodes. Les cercopithèques ont causé la perte de $54 \%$ de la récolte de maïs attendue pour la saison, toutes les parcelles visitées prises en compte et la perte de $18 \%$ de pieds de manioc. Les aulacodes ont détruit $6 \%$ de peds de palmiers plantés.

Pour ce qui est de Yao-akakro, les grandes pertes de cultures ont été du fait des aulacodes Thryonomys swinderianus, des potamochères Potamochoerus porcus, et des Oiseaux* Les aulacodes ont perdu un total de $35,10 \%$ de pieds de maïs pour toutes les parcelles échantillonnées et les potamochères ont provoqué en une seule 
incursion une perte moyenne de $6,6 \%$ de pieds de manioc. Les Oiseaux ont consommé $85 \%$ des grains de maïs semés sur les parcelles échantillonnées du côté de Yaoakakro.

\section{Etendue des dégâts}

Parties des plantes et organes des plantes touchées lors des incursions des animaux

Une même culture peut attirer différentes espèces animales cependant le mode de déprédation se fait différemment par ces espèces qui ont pour chacune, un intérêt particulier pour une culture précise (Tableau 3).

\section{Etendue des pertes de cultures}

Nous avons mesuré les superficies détruites et estimé les coûts de pertes des cultures touchées par les différentes espèces animales impliquées (Tableau 2).

$\mathrm{Au}$ niveau de Kongodjan, sur une surface totale de $12610 \mathrm{~m}^{2}$ de parcelles de maïs, les cercopithèques ont perdu $6856 \mathrm{~m}^{2}$, équivalant à la perte de $274240 \mathrm{~F}$ CFA de revenus des paysans pour un coût global de 504400 F CFA de revient du maïs planté sur les parcelles visitées. Ils ont également ravagé une surface de $3761 \mathrm{~m}^{2}$ de pieds de manioc pour une surface totale de $21350 \mathrm{~m}^{2}$ visitée, cette perte équivaut à 195083 F CFA de pertes de revenus pour un coût de revient des parcelles visitées de 1107424 F CFA.

$\mathrm{Au}$ niveau de Yao-akakro, les aulacodes ont ravagé une parcelle moyenne de $595 \mathrm{~m}^{2}$ de pieds de mais sur une surface de $1696 \mathrm{~m}^{2}$ parcourue, ceci équivaut à 23800 F CFA de perte pour les paysans victimes pour un coût de revient de ces denrées équivalant à $67840 \mathrm{~F}$ CFA. Les prélèvements hors grilles ont permis de relever la perte d'une surface de $233 \mathrm{~m}^{2}$ par les potamochères pour une superficie totale visitée de $3540 \mathrm{~m}^{2}$, ces dégâts valent 12085 F CFA pour un coût de revient des pieds sur les parcelles de 183619 F CFA.

Au niveau de Dohouan, les aulacodes ont détruit une surface de $857,1 \mathrm{~m}^{2}$ sur une surface de $10000 \mathrm{~m}^{2}$ visitée, ce dégât vaut 12000 FCFA pour un coût de revient de la surface repiquée de palmiers de 140000 FCFA. Le Test de Kruskal-Wallis démontre qu'il existe une différence significative entre l'étendue des dégâts de cultures pour chaque espèce impliquée, $\mathrm{Khi}^{2}=25,811$; $\mathrm{ddl}=5$ et $\mathrm{p}<0,001$.

\section{Actions de prévention utilisées}

Les enquêtes ont révélé que parmi les planteurs et les pêcheurs victimes de déprédations sur leurs cultures et autres, $3 \%$ tolèrent les animaux, 33\% parlent d'utilisation de méthodes de protection sans danger pour les animaux. Ces méthodes répertoriées sont : l'entretien des champs (Selon les enquêtés, entretenir le champ, c'est débarrasser régulièrement le champ des mauvaises herbes), la surveillance des champs par la seule présence humaine par les cris et jets de pierres, l'utilisation d'épouvantails, la construction de barrières. $32 \%$ des personnes interrogées utilisent des méthodes dangereuses pour les animaux. Ces méthodes impliquent l'utilisation d'armes à feux, de produits toxiques ainsi que la pose de pièges. Par ailleurs, 32\% restants des enquêtés sont restés sans donner d'avis sur la question.

Par les observations directes sur les parcelles de cultures, nous avons identifié comme moyens utilisés pour les protéger: la pratique de l'entretien des champs; la surveillance, la pose de clôtures, de grillages, de pièges; l'utilisation de produits toxiques et de fusils justifié par la présence de douilles dans les grilles d'observations. 


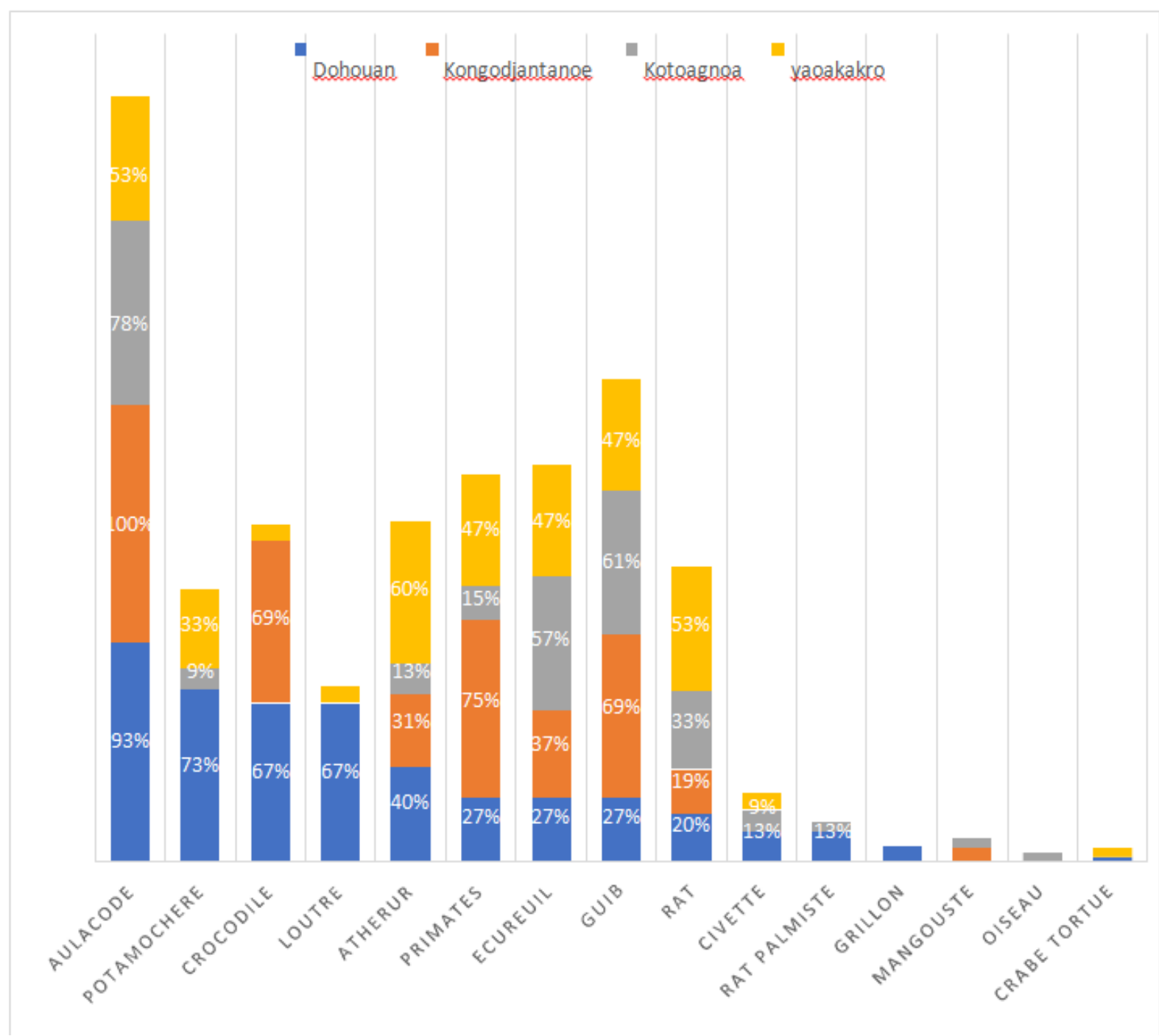

Figure 2: Animaux sauvages impliqués dans les conflits homme faune sauvage après enquête.

Tableau 2: Superficies, proportions (\%) des cultures endommagées et pertes (en franc CFA) occasionnées par différentes espèces animales de la FMTE

\begin{tabular}{|c|c|c|c|c|c|c|c|}
\hline villages & $\begin{array}{l}\text { Espèces } \\
\text { animales } \\
\text { fautives }\end{array}$ & cultures & $\begin{array}{l}\text { Superficies } \\
\text { totales } \\
\text { visitées }\end{array}$ & $\begin{array}{l}\text { Superficies } \\
\text { détruites }\end{array}$ & $\begin{array}{c}\text { Proportion } \\
\text { détruites }\end{array}$ & $\begin{array}{c}\text { Coût } \\
\text { des } \\
\text { pertes }\end{array}$ & $\begin{array}{c}\text { coût de } \\
\text { revient } \\
\text { de la } \\
\text { parcelle }\end{array}$ \\
\hline & & & $\left(\mathrm{m}^{2}\right)$ & $\left(\mathrm{m}^{2}\right)$ & $\%$ & FCFA & FCFA \\
\hline \multirow{4}{*}{ Dohouan } & \multirow{2}{*}{$\begin{array}{l}\text { Atherurus } \\
\text { africanus }\end{array}$} & Maïs & 44350 & 420 & $1 \%$ & 16800 & 1774000 \\
\hline & & Manioc** & 10000 & 20,2 & $0,20 \%$ & 1047 & 518700 \\
\hline & \multirow{2}{*}{$\begin{array}{l}\text { Thryonomys } \\
\text { swinderianus }\end{array}$} & Maïs & 44350 & 1090 & $2,52 \%$ & 43600 & 1774000 \\
\hline & & Manioc** & 10000 & 21,8 & $0,21 \%$ & 1130 & 518700 \\
\hline
\end{tabular}


M. L. KOUAO et al. /Int. J. Biol. Chem. Sci. 12(4): 1717-1730, 2018

\begin{tabular}{|c|c|c|c|c|c|c|}
\hline & palmier & 10000 & 857,1 & $9 \%$ & 12000 & 140000 \\
\hline Oiseaux* & Maïs & 44350 & 157,4 & $0,4 \%$ & 6296 & 1774000 \\
\hline $\begin{array}{l}\text { Francolinus } \\
\text { squamatus }\end{array}$ & Manioc** & 10000 & 9,5 & $0,10 \%$ & 493 & 518700 \\
\hline Manis sp. & Manioc** & 10000 & 0,41 & $0,00 \%$ & 21 & 518700 \\
\hline Cricetomys emini & Maïs & 44350 & 14,5 & $0,03 \%$ & 580 & 1774000 \\
\hline $\begin{array}{l}\text { Tragelaphus } \\
\text { scriptus }\end{array}$ & Manioc** & 10000 & ND & ND & ND & 518700 \\
\hline Hystrix cristata & Maïs & 44350 & 306 & $1 \%$ & 12240 & 1774000 \\
\hline $\begin{array}{l}\text { Atherurus } \\
\text { africanus }\end{array}$ & Manioc** & 21350 & 2 & $0,00 \%$ & 103 & 1107424 \\
\hline $\begin{array}{l}\text { Tragelaphus } \\
\text { scriptus }\end{array}$ & Manioc** & 21350 & ND & ND & ND & 1107424 \\
\hline \multirow{3}{*}{$\begin{array}{l}\text { Thryonomys } \\
\text { swinderianus }\end{array}$} & Maïs & 12610 & 37 & $0,30 \%$ & 1480 & 504400 \\
\hline & Manioc** & 21350 & 11 & $0,05 \%$ & 570 & 1107424 \\
\hline & Palmiers à huile & 10000 & 571,4 & $6 \%$ & 8000 & 140000 \\
\hline Ecureuil* & Cacao & 20000 & 7,6 & $0,04 \%$ & 912 & 1200000 \\
\hline Cricetomys emini & Cacao & 20000 & 0,3 & $0,00 \%$ & 36 & 1200000 \\
\hline \multirow{2}{*}{ Cercopithecus sp. } & Maïs & 12610 & 6856 & $54 \%$ & 274240 & 504400 \\
\hline & Manioc** & 21350 & 3761 & $18 \%$ & 195083 & 1107424 \\
\hline $\begin{array}{l}\text { Atherurus } \\
\text { africanus }\end{array}$ & Cacao & 15000 & 6,3 & $0,04 \%$ & 756 & 1800000 \\
\hline \multirow{3}{*}{$\begin{array}{l}\text { Thryonomys } \\
\text { swinderianus }\end{array}$} & Maïs & 1696 & 595 & $35,10 \%$ & 23800 & 67840 \\
\hline & Manioc** & 45040 & 879,9 & $2,00 \%$ & 45640 & 2336224 \\
\hline & palmiers & 25000 & 785,7 & $3,14 \%$ & 8199 & 350000 \\
\hline Genetta tigrina & Cacao & 15000 & 710 & $4,70 \%$ & 85200 & 1800000 \\
\hline Ecureuil* & Cacao & 15000 & 40 & $0,26 \%$ & 4800 & 1800000 \\
\hline Oiseaux* & Maïs & 1696 & 1434,7 & $85 \%$ & 57380 & 67840 \\
\hline Cricetomys emini & Manioc** & 45040 & 0,4 & $0,00 \%$ & 20 & 2336224 \\
\hline Hystrix cristata & Maïs & 1696 & 18,4 & $1,10 \%$ & 736 & 67840 \\
\hline $\begin{array}{l}\text { Tragelaphus } \\
\text { scriptus }\end{array}$ & Manioc** & 45040 & ND & ND & ND & 2336224 \\
\hline $\begin{array}{l}\text { Potamochoerus } \\
\text { porcus }\end{array}$ & Manioc*** & 3540 & 233 & $6,60 \%$ & 12085 & 183619 \\
\hline
\end{tabular}

Légende: *= Différentes espèces animales du même groupe zoologique non identifiable par leurs traces ;

$\mathrm{ND}=$ Non Disponible, les feuilles de manioc broutées n'ont pas été converties en superficies. 
Tableau 3 : parties et/ou organes attaqué(e)s par les animaux sauvages identifiés sur les cultures par village.

\begin{tabular}{|c|c|c|c|}
\hline Villages & Espèces animales & Cultures & Parties/organes touché(e)s de la plante \\
\hline \multirow{12}{*}{ Dohouan } & Thryonomys swinderianus & \multirow{6}{*}{ Maïs } & Tiges*/grains \\
\hline & Atherurus africanus & & grains \\
\hline & Hystix cristata & & grains \\
\hline & Cricetomys emini & & grains \\
\hline & Cercopithecus sp. & & grains \\
\hline & Oiseaux* & & semis \\
\hline & Atherurus africanus & \multirow{5}{*}{ Manioc** } & Tubercules \\
\hline & Thryonomys swinderianus & & Tiges*/tubercules \\
\hline & Francolinus squamatus & & Tubercules \\
\hline & Manis sp. & & Tubercules \\
\hline & Tragelaphus scriptus & & feuilles \\
\hline & Thryonomys swinderianus & Palmiers à huile & Tiges* \\
\hline \multirow{8}{*}{ Kongodjan } & Thryonomys swinderianus & Mä̈s & Tiges*/grains \\
\hline & Cercopithecus sp. & Nouts & Grains \\
\hline & Atherurus africanus & \multirow{4}{*}{ Manioc** } & Tubercules \\
\hline & Tragelaphus scriptus & & Feuilles \\
\hline & Thryonomys swinderianus & & Tiges*/tubercules \\
\hline & Cercopithecus sp. & & Tiges*/tubercules \\
\hline & Thryonomys swinderianus & Palmiers à huile & Tiges* \\
\hline & $\begin{array}{c}\text { Ecureuil* } \\
\text { Cricetomys emini }\end{array}$ & Cacao & Fèves cacao \\
\hline \multirow{9}{*}{$\begin{array}{c}\text { Yao- } \\
\text { akakro }\end{array}$} & Thryonomys swinderianus & & Tiges*/grains \\
\hline & Oiseaux* & Maïs & semis \\
\hline & Hystrix cristata & & \\
\hline & Thryonomys swinderianus & \multirow{4}{*}{ Manianioc** } & Tiges*/tubercules \\
\hline & Cricetomys emini & & Tubercules \\
\hline & Tragelaphusscriptus & & Feuilles \\
\hline & Potamochoerus porcus & & Tiges* \\
\hline & Thryonomys swinderianus & Palmiers à huile & Tiges \\
\hline & $\begin{array}{c}\text { Genetta tigrina } \\
\text { Ecureil }^{*}\end{array}$ & cacao & Fèves cacao \\
\hline
\end{tabular}




\section{DISCUSSION}

L'enquête a montré qu'il existe trois différentes formes de conflits entre les hommes et les animaux autour de la FMTE: les déprédations de cultures, les dégâts sur le matériel de pêche et la consommation par les animaux des poissons qui s'y trouvent puis, la réaction des hommes envers les animaux sauvages. Parmi ces trois formes, les déprédations sur les cultures sont les plus souvent rencontrées, démontré également par Hill et al. (2004), Les enquêtes n'ont pas révélé d'attaques animales sur les populations autour de cette forêt contrairement à d'autres pays où il existe ce genre d'attaques sur les hommes occasionnant malheureusement des morts d'hommes (FAO, 2010).

En confrontant les résultats issus des enquêtes à ceux des observations directes, nous notons qu'à l'instar d'autres travaux, plusieurs espèces animales sont impliquées dans les dégâts de cultures avec une unanimité sur l'aulacode qui engendre quasiment sur toutes les parcelles des dégâts (Drazo et al., 2008). Nous notons cependant que contrairement aux incursions côté forêt dégradée dominées par les rongeurs, du côté de la forêt dense, les cercopithèques, les potamochères font des incursions dans les champs et occasionnent de grandes pertes de cultures. Ceci présage d'une bonne conservation de la FMTE de ce côté de la FMTE telle que démontré par Nyirenda (2013). Suivant les résultats de leurs travaux, les hommes installés autour des forêts bien conservées souffrent plus des ravages des animaux. Même si selon les proportions, beaucoup d'enquêtés accepte que les animaux se nourrissent sur leurs cultures, nous relevons que le mode de prélèvement adopté par certains animaux sur les cultures, expose à l'insécurité alimentaire. Les aulacodes causent d'énormes pertes aux cultivateurs de manioc en détruisant de nombreux pieds de manioc ou de maïs non matures pour des raisons non élucidées dans ce travail. Une raison peut être trouvée dans les résultats des travaux de Eniang et al. (2011). Ils ont prouvé que les singes Chlorocebus tantalus ont une répugnance pour les tubercules d'une certaine variété de manioc cultivée. Les cercopithèques en quête de tubercules, causent d'énormes pertes aux paysans en déterrant les pieds de manioc, même les plus jeunes à l'instar des potamochères, identifiés comme des animaux à problèmes (Kagoro Rugunda, 2004). Les oiseaux du côté de Yao-akakro ont découragé les paysans en consommant la quasi-totalité des grains semés, ils n'en ont plus planté craignant la même invasion.

En vue de se prémunir des dégâts, deux formes de méthodes sont pratiquées par les paysans, les méthodes traditionnelles sans danger pour les animaux et les méthodes avec risques pour ces derniers. Les animaux sont ainsi exposés aux pièges placés par les paysans ou par les produits toxiques pulvérisés par les paysans dans les champs quand ils ne sont pas abattus par ceux-ci. Les produits toxiques utilisés sont surtout les herbicides qu'ils emploient abusivement en même temps pour éliminer les mauvaises herbes et repousser selon eux les animaux sauvages. D'après Hill (2000), les moyens utilisés par les populations pour se prémunir des attaques ou les régler sont potentiellement révélateurs des représentations que se font celles-ci à l'égard des animaux sauvages. Malheureusement, les méthodes utilisées ne sont toujours pas à la mesure de leurs espérances puisque les incursions animales sur les parcelles ne se sont estompées définitivement après la pratique de celles-ci. Il est à relever pour le compte des gestionnaires de la FMTE que diverses méthodes d'atténuations ont été développées pour venir en aide aux agriculteurs victimes des intrusions des animaux (Hoare, 2012). Ces méthodes souffrent $\mathrm{du}$ problème d'accommodation et deviennent moins efficaces avec le temps (Naughton-Treves, 1997), pour cela, leur mise en essai perpétuelle est faite afin d'adopter les plus efficaces. Il est à noter qu'aucune de ces méthodes n'a été retrouvée autour de la FMTE.

\section{Conclusion}

Les espèces animales occasionnent des pertes de cultures plus ou moins importantes aux paysans installés à la limite de la FMTE. Parmi elles, les aulacodes Thryonomys swinderianus et les cercopithèques Cercopithecus sp., constituent de loin les plus grands ravageurs.

Dans un souci de maintien de la sécurité alimentaire pour une part et pour la conservation durable des animaux sauvages d'autre part, il s'avère opportun de tester les méthodes de gestion proposées et pratiquer 
ailleurs telles que suggérées par la FAO. Ceci vaudra à longs termes de mettre en place des mesures et méthodes de gestions durables de ce type de conflits autour de la FMTE.

\section{CONFLITS D'INTERETS}

Les auteurs déclarent qu'ils n'ont pas de conflit d'intérêts pour cet article.

\section{CONTRIBUTIONS DES AUTEURS}

Tous les co-auteurs ont contribué à la collecte des données, à l'identification des animaux déprédateurs et à la relecture de versions antérieures de cet article.

\section{REMERCIEMENTS}

Nous remercions le Ministère de l'Enseignement Supérieur et l'UFR Biosciences à l'Université Félix Houphouet Boigny pour avoir autorisé cette étude. Nous remercions également le programme RASAPCI (Recherches et Actions pour la Sauvegarde des Primates en Côte d'Ivoire) pour la formation et la reconversion des chasseurs expérimentés en prospecteurs faune. Nous remercions enfin le CSRS (Centre Suisse de Recherches Scientifiques en Côte d'Ivoire) pour avoir facilité cette étude.

\section{REFERENCES}

Atta AC, Soulemane O, Yao K A, Kasse KB, Yaokokore-Behibro KH. 2016. Caractérisation des conflits hommeelephant dans le departement de sikensi (sud-est Côte d'Ivoire). Agronomie Africaine, 28(3) : 30 - 41.

Bene J-CK, Kone I, Gonedele Bi S, Bitty EA, Ouattara K, Akpatou KB, N'guessan KA, Koffi DA. 2012. The diurnal primate community of the Tanoé Forest: species composition, relative abundance, distribution, polyspecific associations and conservation status. International Journal of Biological and Chemical Sciences, 6(1): 51-64. DOI: http://dx.doi.org/10.4314/ijbcs.v6i1.5

Bulte E, Rondeau D. 2007. Compensation for wildlife damages: Habitat conversion,species preservation and local welfare. Journal of Environmental Economics and Management, 54: 311322. Doi:10.1016/j.jeem.2007.02.003.
Drazo N. A, Kennis J, Leirs H, Migimiru D. A. 2008. Farmer survey in the hinterland of Kisangani (Democratic Republic of Congo) on rodent crop damage and rodent control techniques used. Mammalia, 72: 192-197. DOI:

10.1515/MAMM.2008.034

Enbakom HW, Feyssa DH, Takele S. 2017. Impacts of deforestation on the livelihood of smallholder farmers in Arba Minch Zuria Woreda, Southern Ethiopia. Afr. J. Agric. Res., 12(15): 1293-1305. DOI: http://dx.doi.org/10.5897/AJAR2015.101

Eniang EA, Ijeomah HM, Okeyoyin G, Uwatt AE. 2011. Assessment of Human Wildlife Conflicts in Filinga Range of Gashaka Gumti National Park, Nigeria. PAT); 7(1): 15-35 ISSN: 0794-5213. http://patnsukjournal.net/Vol7No1/p2.pdf

Fairet EMM. 2012. Vulnerability to cropraiding: an interdisciplinary investigation in Loango National Park, Gabon. PhD thesis, Durham University, p 218.

FAO. 2010. Les conflits humains-faune en Afrique: Causes, conséquences et stratégies de gestion. FAO; P 110.

Faye B, Bassene C, Camara AA, Ngom A, Mbaye MS, Noba K. 2018. Flore et végétation de la Réserve Spéciale de Faune de Gueumbeul (Sénégal). Int. J. Biol. Chem. Sci., 12(1): 43-61. DOI: https://dx.doi.org/10.4314/ijbcs.v12i1.4

Hill CM. 2000. Conflict of interest between people and baboons: crop raiding in Uganda. International Journal of Primatology, 21(2): 299-315. http://citeseerx.ist.psu.edu/viewdoc/down load?doi=10.1.1.470.9 $835 \&$ rep $=$ rep $1 \&$ type $=$ pdf.

Hill C. 2004. Farmers' Perspectives of Conflict at the Wildlife-Agriculture Boundary: Some Lessons Learned from African Subsistence Farmers. Human Dimensions of Wildlife, 9: 279-286. DOI: 10.1080/10871200490505710

Hoare RE. 2012. Lessons from 15 years of human-elephant conflict mitigation: Management considerations involving biological, physical and governance issues in Africa. Pachyderm, 51: 60-74. https://Doi.org/10.1080/10871209.2015. 1005855 . 
Kagoro-Rugunda G. 2004. Crop raiding around Lake Mburo National Park, Uganda. African Journal of Ecology, 42: 32-41.

https://onlinelibrary.wiley.com/Doi/full/ 10.1111/j.0141-6707.2004.00444.x

Koné I, Gléanou KE, Koffi DA, Agnissan A, Zadou DA, Ibo GJ. 2012. Mobilisation sociale inédite pour sauver une forêt du domaine rural dans le Sud-Est de la Côte d'Ivoire: le rôle des logiques socioculturelles. Les Services Culturels, Sociaux et Spirituels de la Forêt.

Kouelo FA, Houngnandan P, Dedehouanou H, Tossou R, Orou Bello DO, Joël Bekou KA, Tchetangni AY. 2016. Soil conservation practices in three watersheds of Benin: Farmers ${ }^{\text {ee }}$ cropping systems characterization. Afr. J. Agric. Res., 11(7): 507-515. DOI: http://dx.doi.org/10.5897/AJAR2015.102 77

Kpan F. 2011. Peuplement des amphibiens anoures de la Forêt des Marais Tanoé-Ehy (Sud-Est Côte d'Ivoire), Mémoire de DEA, UCA/CSRS, p 63.

Marchand G. 2013. Les conflits hommes/animaux sauvages sous le regard de la géographie. Cadre territorial, perceptions et dimension spatial. Carnets de géographes, $\mathrm{n}^{\circ} 5$.

MINEF (Ministère des Eaux et Forêts). 2015. Atelier de réflexion sur les conflits home faune sauvage, République de Côte d'Ivoire.

Naughton-Treves, L. 1997. Whose Animals? A history of property rights to wildlife in Toro, western Uganda. Land Degradation and Development, 10: 311-328. https://onlinelibrary.wiley.com/Doi/abs/1 0.1002/(SICI)1099145X(199907/08)10:4 \%3C311::AID-LDR362\%3E3.0.CO;2-3

Naughton-Treves, L., 1998. Predicting patterns of crop raiding by wildlife around Kibale National Park, Uganda. Conserv. Biol., 12: 156-168.

Nyirenda VR, Myburgh WJ, Reilly BK, Phiri AI, Chabwela HN. 2013. Wildlife crop damage valuation and conservation: conflicting perception by local farmers in the Luangwa Valley, eastern Zambia. International Journal of Biodiversity and
Conservation, 5(11): 741-750. DOI: 10.5897/IJBC12.026

Parker GE, Osborn FV, Hoare RE, Niskanen LS. 2007. Human-elephant conflict mitigation: a training course for community-based approaches in Africa. Participant's manual. Livingstone, Zambia, Elephant Pepper Development Trust and Nairobi, Kenya, IUCN Species Survival Commission, African Elephant Specialist Group, Human-Elephant Conflict Task Force.

Pupion P-C. 2008 Statistiques pour la gestion, Applications Excel et SPSS, 2ème édition.

Sidawy P. 2011. Etat des lieux des conflits hommes-faune sauvage autour de la Forêt des Marais Tanoé-Ehy en Côte d'Ivoire. Master Biologie, Université Montpellier2 Géosciences, Agronomie, Environnement Spécialité Ecologie fonctionnelle et développement durable, P 23.

WWF. 2005. Human Wildlife Conflict Manual. Wildlife Management series.

Ye L, Lata JC, Masse D, Nacro HB, Sébastien Barot S. 2017. Effets du pâturage sur la biomasse herbacée et sur des paramètres chimiques et biologiques des sols dans une savane arbustive au Burkina Faso. Int. J. Biol. Chem. Sci., 10(6): 2539-2554. DOI: http://dx.doi.org/10.4314/ijbcs.v10i6.11.

Zadou D, Kone I, Mouroufié VK, Adou YYC, Gleanou EK, Kablan YA, Coulibaly D, Ibo JG. 2011. Valeur de la forêt des Marais Tanoé-Ehy (sud-est de la COTE D'IVOIRE) pour la conservation: dimension socio-anthropologique. Mongabay.com Open Access Journal Tropical Conservation Science, 4(4): 373-385.

Zoumarou NW, Bagnan MA, Akossou AYY, Kanlindogbe CB. 2016. Caractérisation morphologique $\mathrm{d}^{\text {ee }}$ une collection de fruits $\mathrm{d}^{\text {ee }}$ anacardier provenant de la commune de Parakou (Bénin). Int. J. Biol. Chem. Sci., 10(6): 2413-2422. DOI: http://dx.doi.org/10.4314/ijbcs.v10i6.1 